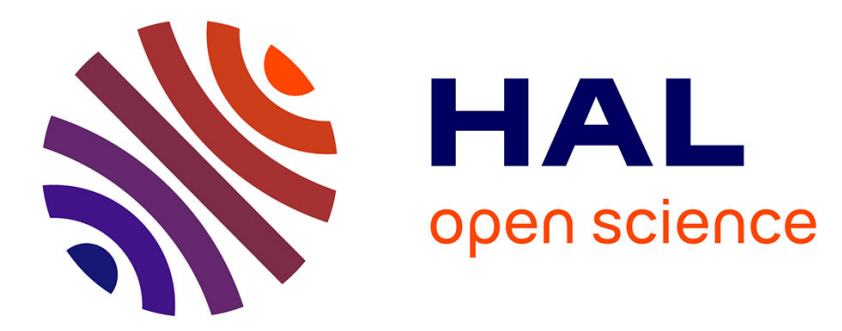

\title{
Homogenization of sound hard metamaterials in the time domain
}

\author{
A Maurel, J.-J Marigo, B Lombard
}

\section{To cite this version:}

A Maurel, J.-J Marigo, B Lombard. Homogenization of sound hard metamaterials in the time domain. 10th International Congress on Advanced Electromagnetic Materials in Microwaves and Optics Metamaterials 2016, Sep 2016, Hellas, Crête, Greece. hal-01322320

\section{HAL Id: hal-01322320 https://hal.science/hal-01322320}

Submitted on 30 May 2016

HAL is a multi-disciplinary open access archive for the deposit and dissemination of scientific research documents, whether they are published or not. The documents may come from teaching and research institutions in France or abroad, or from public or private research centers.
L'archive ouverte pluridisciplinaire HAL, est destinée au dépôt et à la diffusion de documents scientifiques de niveau recherche, publiés ou non, émanant des établissements d'enseignement et de recherche français ou étrangers, des laboratoires publics ou privés. 


\title{
Homogenization of sound hard metamaterials in the time domain
}

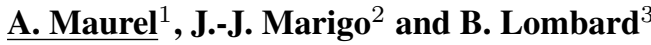 \\ ${ }^{1}$ I. Langevin, 1 rue Jussieu, Paris 75005, France, \\ ${ }^{2}$ LMS, Ecole Polytechnique, Route de Saclay, 91128 Palaiseau, France \\ ${ }^{3}$ LMA, 4 impasse Nicolas Tesla, 13453 Marseille Cedex 13, France \\ agnes.maurel@espci.fr
}

\begin{abstract}
We present a homogenization method based on a matched asymptotic expansion technique for sound hard materials structured at subwavelength scale. Considering the wave equation in the time domain, jump conditions are derived for the acoustic pressure and the normal velocity across an equivalent interface with non zero thickness. These jump conditions are implemented in a numerical scheme and compared to the results of the direct problem.
\end{abstract}

\section{INTRODUCTION}

Since Pendry's works [1], acoustic metamaterials consisting in massive materials perforated by periodic subwavelength holes have been shown to be able to control the wave propagation with high flexibility. However, if the metamaterials have a subwavelength period, many of the observed phenomena are attributable to Fabry-Perot type resonances, which requires the thickness of these metamaterials to be at wavelength scale (and the thickness refers to the size in the direction perpendicular to the planes containing the periodic cells). In order to reduce the size of the devices, structures with a subwavelength thickness have been developed, and they are known as metasurfaces and metafilms. Because of their subwavelength periodicity, homogenization techniques are natural tools to describe the effective properties of metamaterials, but the vanishing thickness of these devices has to be accounted for. We present an interface homogenization following the approach developed in solid mechanics [2], and that we adapt to the wave equation in the time domain. Equivalent jump conditions are obtained across an equivalent interface, which allows to define a (positive) energy supported by this interface. A numerical implementation of the homogenized problem is proposed and the results are compared with results of the real problem.

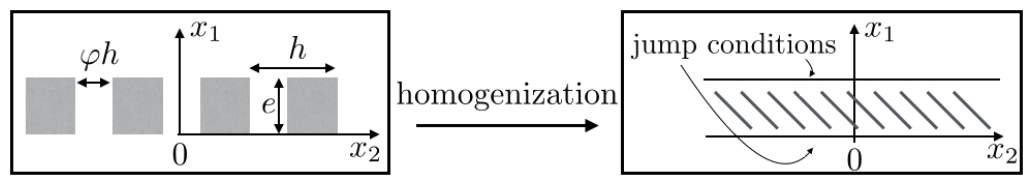

Fig. 1: Array of periodic rigid inclusions; the homogenization gives an equivalent thin interface associated to jump conditions, Eqs. (2).

\section{EFFECTIVE JUMP CONDITIONS AND ENERGY OF THE EQUIVALENT INTERFACE}

\section{A. Effective jump conditions}

We consider the linearized Euler equations for the acoustic pressure $P(\mathbf{x}, t)$ and velocity $\mathbf{U}(\mathbf{x}, t)$ in the time domain, with $t$ the time and $\mathbf{x}=\left(x_{1}, x_{2}\right)$ the spatial coordinates

$$
\rho_{0} \frac{\partial \mathbf{U}}{\partial t}=-\nabla P, \quad \chi_{0} \frac{\partial P}{\partial t}+\operatorname{div} \mathbf{U}=0,
$$

( $\rho_{0}$ is the mass density, $\chi_{0}=\left(\rho_{0} c_{0}^{2}\right)^{-1}$ is the isentropic compressibility of the fluid with $c_{0}$ the sound speed in the fluid). We consider acoustic waves with a minimum wavelength $2 \pi / k$ larger than the typical periodicity of the microstructuration $h$, such that $\varepsilon \equiv k h$ is a small parameter. 
The effective jump conditions are obtained (at second order in $\varepsilon$ ) from a matched asymptotic expansion as presented in [3]. The derivation is based on a separation of the space into the far field and the near field and a two scale expansion of the near field solution. The near field solution can be expressed in terms of two elementary functions, being themselves solutions of static problems (which depend only on the geometry of the microstructure). Next, the two, far field and near field, solutions are matched asymptotically, leading to jump conditions for the far field solution across the near field region being considered as an equivalent interface (afterwards this region is not considered). The jump conditions at the equivalent interface of thickness $e$ read

$$
\left\{\begin{array}{l}
{[P]=h \mathcal{B} \frac{\overline{\partial P}}{\partial x_{1}}} \\
{\left[U_{1}\right]=e \varphi \frac{\overline{\partial U_{1}}}{\partial x_{1}}+h \mathcal{C} \frac{\overline{\partial U_{2}}}{\partial x_{2}}}
\end{array}\right.
$$

with $(\mathcal{B}, \mathcal{C})$ two parameters defined by the elementary problems. For rectangular sound hard inclusions, explicit expressions are

$$
\mathcal{B}=\frac{e}{h \varphi}+\frac{2}{\pi} \log \left(\sin \frac{\pi \varphi}{2}\right)^{-1} . \quad \mathcal{C} \simeq \frac{e}{h} \varphi-\frac{\pi}{8} \varphi^{2},
$$

The complete derivation of these conditions can be found in $[3,4]$. The notation $[f]$ refers to the $f(e)-f(0)$ (Fig. 1 ) being the jump of the field $f$ across the equivalent interface and $\bar{f}=(f(0)+f(e)) / 2$.

\section{B. Energy conservation for the equivalent thin interface}

In a bounded domain $\Omega$ of the real problem, energy conservation reads

$$
\frac{d}{d t} \int_{\Omega} d V\left(\frac{\rho_{0}}{2} U^{2}+\frac{\chi_{0}}{2} P^{2}\right)+\int_{\Sigma} d S P \mathbf{U} \cdot \mathbf{n}=0
$$

with $\Sigma=\partial \Omega$. Let us write the equation of energy conservation in the homogenized problem. In this problem, the domain occupied by the microstructuration is not considered (numerically, this means that this domain $\Omega_{e}$ will not be resolved). Now the boundary $\partial \Omega$ includes the boundaries of $\Omega_{e}$, from which the equation of energy conservation is

$$
\frac{d}{d t} \int_{\Omega \backslash \Omega_{e}} d V\left(\frac{\rho_{0}}{2} U^{2}+\frac{\chi_{0}}{2} P^{2}\right)+\frac{d}{d t} \mathcal{E}_{e}+\int_{\Sigma} d S P \mathbf{U} . \mathbf{n}=0,
$$

where $\mathcal{E}_{e}$ is the energy supported by the equivalent interface, which takes the form (from the jump conditions, Eqs. (2))

$$
\mathcal{E}_{s}=\int \mathrm{d} X_{2}\left[\frac{\rho_{0}}{2}\left(h \mathcal{B}{\overline{U_{1}}}^{2}+(e \varphi-h \mathcal{C}){\overline{U_{2}}}^{2}\right)+\frac{\chi_{0}}{2} e \varphi \bar{P}^{2}\right],
$$

and this energy is positive (with $\mathcal{B}>0$ and $e \varphi<h \mathcal{C}$ ), which is suitable for numerical purpose.

\section{NUMERICAL VALIDATION}

The time-domain simulations are done on a regular Cartesian grid, with a numerical scheme (ADER 4) being fourth-order accurate both in space and time [5]. The interface conditions (being of Neumann type for the real problem and being the effective conditions, Eqs. (2) in the homogenized problem) are discretized by an immersed interface method [6]. We report the results obtained for a microstructure made up of rectangular sound hard inclusions periodically located along a line $\left(x_{1}=0\right) ; e / h=1$ and $\varphi=0.5$ have been considered. The homogenized version of this microstructure is an enlarged interface which occupies the same domain as the microstructure (and $\mathcal{B}=2.22$ and $\mathcal{C}=0.42$ ). Fig. 2 show typical instantaneous pressure fields after the reflection and transmission of an incident wave on the microstructure. The incident wave has been generated by a punctual source (a truncated sinusoid with central frequency associated to a dimensionless wavenumber $k h ; k h=0.84$ and $k h=1.25$ have been considered). The difference between the real field and its homogenized version is reasonable for $k h=0.84$ $(8 \%)$ and becomes visible for $k h=1.25(20 \%)$; nevertheless, it is remarkable that these limiting cases of $k h$ close to unity do not produce a complete fail of the homogenization predictions. 

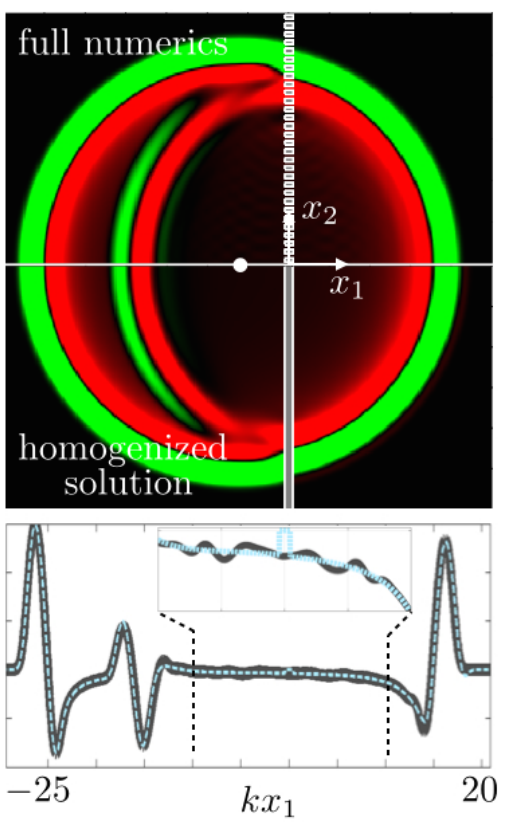
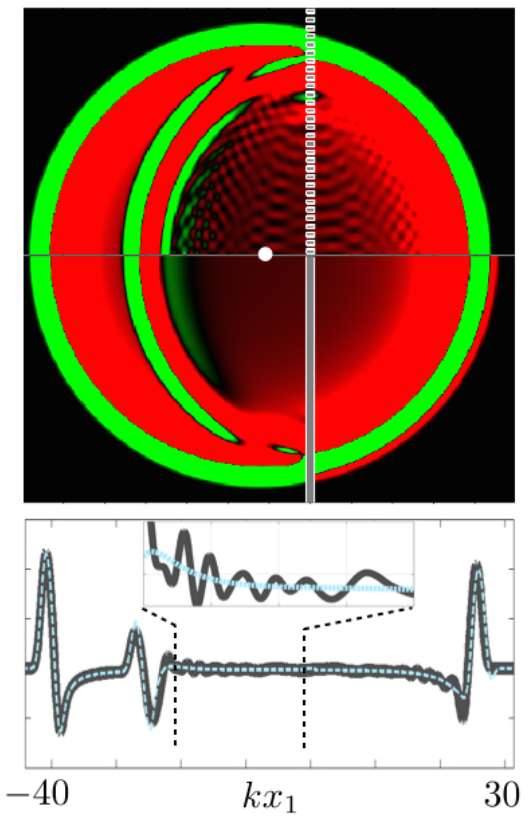

Fig. 2: Top panels: Instantaneous pressure fields obtained after the reflection and the transmission of the signal emitted by a source point (white point); the upper and lower half-spaces show the fields obtained by solving the full problem and by solving the homogenized problem (left for $k h=0.84$ and right for $k h=1.25$ ). Bottom panels: pressure along the $x_{2}=0$-line, for the full problem (grey lines) and for the homogenized problem (dotted lines); the insets show a zoom of the coda.

The reflected and transmitted waves are characterized by a main "ballistic" pulse followed by a long coda-type signal due to multiple reflections within the microstructure. If the continuities of the pressure field and of the normal velocity were assumed (leading order approximation, or order 1), only the ballistic part of the signal would be recovered, with an error due to an overestimate of the energy transported by the ballistic waves. With the jump conditions written at order 2 in Eq. (2), the main characteristic of the coda is captured, and main characteristic is meant here notably in terms of the energy supported by the coda.

\section{CONCLUSION}

The capability of our jump conditions to capture the behavior of microstructured films can be used to predict the properties of metamaterials. For instance, many of the so-called metasurfaces correspond to the same geometry as in Fig. 2 with a rigid plate at $x_{1}=d$; this configuration is known to produce resonances at the subwavelength scale which can be tuned to produce efficient metascreens.

\section{REFERENCES}

[1] J.B. Pendry, L. Martin-Moreno and F.J. Garcia-Vidal, "Mimicking surface plasmons with structured surfaces", Science, vol. 5685, p. 847-848, 2004.

[2] J.-J. Marigo, C. Pideri, "The effective behavior of elastic bodies containing microcracks or microholes localized on a surface", Int. J. Damage Mech., p. 1056789511406914, 2011

[3] J.-J. Marigo and A. Maurel, "An interface model for homogenization of acoustic metafilms" DOI: 10.13140/RG.2.1.3667.7206, submitted, 2016.

[4] J.-J. Marigo and A. Maurel, "Homogenization models for thin rigid structured surfaces and fims", submitted to JASA, 2016.

[5] G. Chiavassa, B. Lombard, "Time domain numerical modeling of wave propagation in 2D acoustic / porous media", Comp. Phys., vol. 13, p. 985-1012, 2013.

[6] B. Lombard, J. Piraux, C. Gélis, J. Virieux, "Free and smooth boundaries in 2-D finite-difference schemes for transient elastic waves", Geophys. J. Int., vol. 172, p. 252-261, 2008. 